natural and most economical means". As a corollary and an amplification he added: "Highway construction leads its engineers into Nature-Nature under all her aspects. For roads are meant to overcome Nature, but at the same time they should be in harmony with Nature. Above all, roads must essentially be built with the material the surrounding Nature provides. We may add bitumen, tar, cement, but as basic material we have to use the stone, the sand, the earth that lies nearby."

It was no accident, therefore, that the foundation stone itself was not the usual marble slab but a rough quartzite stone dug out of the very site. With a feeling of pride, Dr. Zipkes said: "We dug it out of our own ground, very near this place. And we brought it here for our foundation stone ceremony to be a symbol of what we are going to do inside the walls that will rise around this stone. Use the materials that Nature provides, and search for ways to construct with them the most solid, safest and most economical roads."

\section{PAINTS AND OTHER SURFACE COATINGS}

$\mathrm{T}$ HE results of much new scientific and technological work relating to paints and other surface coatings and printing inks, including new raw materials and equipment, were displayed at the third Technical Trade Exhibition organized by the London Section of the Oil and Colour Chemists' Association and held at the Borough Polytechnic, London, during March 19-21. The number of exhibitors increased from twenty-six in 1950 to thirty-four this year.

The Paint Research Station demonstrated an approach to the prediction of the colour of pigment mixtures, and some applications of radioactive tracers. The radioactive isotope in natural potassium has been used for determining this element in potassium chromes ; and pigment sedimentation, flotation, and drier absorption are other problems to be investigated by the new techniques. The mechanism of polymerization will be studied by introducing radioactive carbon into different parts of the molecules of drying oils.

A photoelectric colour meter was shown which has eight colour-filters stopped down so that the same amount of light passes through each; one setting for 100 per cent (with magnesium oxide) then suffices for a series of panels. Another exhibit, a prototype of a reflecting microscope, showed the instrument to be of simple design and very easily adjusted. A system of mirrors projects the image (free from chromatic aberration) on a grey screen, and there is no eyepiece. Modifications under development include the use of downward illumination for examining opaque objects.

Attractive items for the laboratory were an unbreakable aluminium vacuum desiccator with plate-glass inspection window and grease-free sealing joint, and a metal tap for ordinary glass vacuum desiccators. The only vacuum oven made in Great Britain with single-action door elosure was also to be seen. A new $Z$-blade mixer that was on show has interchangeable 1-pint and 1-quart pan sections and blades, and a heavy-duty stainless steel mixer in three sizes has a new type of packed gland and can be adapted for working under vacuum.
Further research and development work on isomerized rubber has led to new applications in printing inks and a new floor paint for concrete, an emulsion providing a vehicle for alkali-resistant wall primers, and a copolymer with styrene for use in waterresistant and anti-corrosive paints. A new finely dispersed polyvinyl acetate emulsion was demonstrated to yield more glossy films than older types and to possess better adhesion after immersion in water. Newly developed polyacrylate dispersions were shown in leather finishes. A new technique gives alkyd resin dispersions of smaller particle-size than formerly, suitable for use in industrial paints.

Epichlorhydrin alkyd resin coatings, stoved or airdried, were seen to resist attack by 70 per cent sulphuric acid and 30 per cent caustic soda, while the remarkable heat resistance of silicone-modified alkyds was exemplified by pigmented finishes stated to have been exposed at $450^{\circ} \mathrm{C}$. for $500 \mathrm{hr}$. and at $300^{\circ} \mathrm{C}$. for $1,000 \mathrm{hr}$., which were without apparent deterioration, except some loss of gloss. Alkydmelamine resins plasticized with coconut oil were shown to possess enhanced colour retention.

Epichlorhydrin-diphenylolpropane resins, a rocently discovered class having only $\mathrm{C}-\mathrm{C}$ and ether bonds, contain reactive hydroxyl and epoxy groups; their particularly good resistance to strong alkali was demonstrated. They can be used cold-cut with a cross-linking resin (for example, urea or melamine), or esterified with drying-oil fatty acids to give airdrying media.

Many quite new derivatives of sebacic acid were exhibited, including diallyl sebacate recommended as a cross-linking agent for drying oils, polypropylene sebacate as a non-migrating plasticizer, sebacamide as a urea resin modifier, and sebacic polyanhydride as an alkyd intermediate. A series of chlorine-containing plasticizers for cellulose acetate comprises trichlorethylideneglycerol and derivatives. A new 'sequestering' agent, the di-sodium salt of ethylene diamine tetra-acetic acid, can deactivate the ions of various metals, and may be used to prevent 'poisoning', decomposition, etc., caused by traces of metal ions, to stabilize emulsions, to titrate metals, and for many other purposes.

Two new adipates, the dinonyl and di(3,5,5-trimethylhexyl) compounds, were shown as lowtemperature plasticizers for cellulose derivatives and resins, and two new phthalate plasticizers for lacquers are the di(2-butoxyethyl) and di(ethylene glycol monobutyl ether) compounds. New high-boiling solvents for non-blushing nitrocellulose lacquer emulsions are octyl acetate (b.p. $198^{\circ}$ C.) and nonyl acetate (b.p. $207^{\circ}$ C.).

Calcium plumbate is a new, light-coloured pigment for anti-corrosive paints; a pale dull tan, it can be tinted green, light stone, grey or brown. The known anti-corrosive property of zinc dust was related in a demonstration to the electrical conductivity of coatings made with this pigment. Interesting panels showed new work with zinc dust in inorganic media such as sodium silicate; in 'cementiferous' com. positions based on barium chloride, potassium dihydrogen phosphate and magnesium chloride; and mixed with zinc oxide in a wood primer. Research on zinc oxide pigments shows that flow and resistance to 'chalking' increase with particle size, while oil absorption falls; under ultra-violet radiation the more reactive particles can be distinguished from the less reactive by the colour of their fluorescence.

NeIL R. FIsk 